\title{
Community-based Oral Health Education Program in a Rural Population of Haryana: A 25 years Experience
}

\author{
${ }^{1}$ Ashima Goyal, ${ }^{2}$ Krishan Gauba, ${ }^{3}$ Utkal Mohanty, ${ }^{4}$ Amrit Tewari
}

\begin{abstract}
Background: An Indian Council of Medical Research (ICMR) task force project was started in 1985 covering a population of 120,000 of Raipur Rani block of Haryana to study the feasibility of implementation of oral health promotion and prevention in the community and in the schools by utilizing existing manpower at different sectors.
\end{abstract}

Objectives: (i) To evaluate the long-term role of healthcare workers in imparting primary preventive strategies of oral health to adult community (ii) To study the knowledge, attitude and practice of the community regarding oral health.

Methodology: A total of 600 households (300 in experimental block and 300 in control block) were included by stratified random sampling method depending on the distance from Community Health Centre of Raipur Rani to assess KAP and Caries activity among the population.

Results: The use of toothbrush as an oral hygiene method is being practiced by $96.6 \%$ of population in the experimental area compared to $84 \%$ in the control population where no oral health promotion activity was carried out. A great variation was seen in the frequency of its usage; $56 \%$ of the population in experimental area brushes twice per day compared to $7 \%$ of control area. According to the present data, $80 \%$ of the population in the experimental area is aware about the etiology, progress and consequences of gum diseases due to continuous oral health education delivered by the trained health staff during their routine beat program. In the control area where no oral health program was implemented, this knowledge was seen in 22 to $35 \%$ of the population.

Conclusion: In a developing country like India there is a pressing need of community-based oral health programs to reduce the burden of oral diseases, improve quality of life and reduce

\footnotetext{
${ }^{1}$ Professor, ${ }^{2}$ Professor and Head

${ }^{3}$ Ex-Senior Resident, ${ }^{4}$ Emeritus Professor

${ }^{1,4}$ Department of Oral Health Sciences Centre, Postgraduate Institute of Medical Education and Research, Chandigarh, India

${ }^{2}$ Department of Pedodontics and Preventive Dentistry, Chair Oral Health Sciences Centre, Postgraduate Institute of Medical Education and Research, Chandigarh, India

${ }^{3}$ Department of Community and Preventive Dentistry, Oral Health Sciences Centre, Postgraduate Institute of Medical Education and Research, Chandigarh, India

Corresponding Author: Ashima Goyal, Professor, Department of Oral Health Sciences Centre, Postgraduate Institute of Medical Education and Research, Chandigarh, India, Phone: 01722756833, e-mail: ashimapgi@yahoo.in
}

out of pocket expenditure incurring toward treatment of these diseases.

Keywords: Caries, Caries activity, Oral health promotion, Oral hygiene, Quality of life.

How to cite this article: Goyal A, Gauba K, Mohanty U, Tewari A. Community-based Oral Health Education Program in a Rural Population of Haryana: A 25 years Experience. J Postgrad Med Edu Res 2015;49(3):101-104.

Source of support: Funded by Indian Council of Medical Research.

Conflict of interest: None

\section{INTRODUCTION}

In a developing nation like India the burden of noncommunicable diseases including oral diseases are on rise in addition to the existing burden of communicable diseases. ${ }^{1}$ Common oral diseases like dental caries (50-60\%) and periodontitis (80-90\%) affect majority of the population ${ }^{2}$ and also share common risk factors with chronic diseases like CVD, diabetes, etc. ${ }^{3-5}$ Oral diseases are expensive to treat and consume a lot of clinical time. In our country where oral healthcare facilities in public health set up are either nonexistent or existing with a very limited spectrum of services, majority of the population receives treatment by out of pocket expenditure. In the above vignette it is imperative to strengthen communitybased oral health promotion to prevent and reduce the burden of oral diseases. Therefore, an ICMR task force project was started in 1985 covering a population of 120,000 of Raipur Rani Block of Haryana (Experimental Area, EA) to study the feasibility of implementation of oral health education in the community through trained health workers. The Sidhaura block was taken as Control Area (CA) where no oral health program was implemented by the investigators. In 1987, the project staff trained the existing health workers, health assistants, multipurpose workers of $\mathrm{CHC}$ of the experimental area in primary preventive strategies of oral health, who further imparted lectures on oral health to the community on a regular basis making oral health education a part and parcel of their routine program. The health workers were given training manuals for their reference and standardized oral health education material for training the community during their routine beat program. The project staff was withdrawn from the field area in 1990 and subsequently 
the health workers were made responsible for delivering lectures to community on a routine basis. Since then the project has been running by self rhythm, being continuously monitored by faculty and a social worker of the oral health sciences centre, PGIMER.

\section{Objectives}

- To evaluate the long-term role of healthcare workers in imparting primary preventive strategies of oral health to adult community as a result of training imparted to them by trainers (dentists).

- To study the present knowledge, attitude and practice (KAP) of the community regarding oral health as a result of implementation of primary preventive oral health education program through multipurpose workers almost 25 years ago and also comparison with the baseline values at that time.

- To compare the knowledge, attitude and practice of adult community of the experimental and control areas to assess the long-term effectiveness/role of health workers in imparting oral health education to adult community during their routine beat program in the experimental area.

\section{METHODOLOGY}

The KAP survey was carried out in the community to elicit information on their knowledge, attitude and practice on the various aspects of oral health. A total of 600 households (300 in experimental block and 300 in control block) were included with stratified sampling design. The stratification was carried out on the basis of distance from community health center ( $\mathrm{CHC}$ ). Three strata were taken viz. $\leq 5 \mathrm{~km}, 5$ to $10 \mathrm{~km}$ and $>10 \mathrm{~km}$ from the $\mathrm{CHC}$ of the experimental and control blocks. A total of 100 households belonging to 4 to 5 villages were included per strata i.e. 100 from $\leq 5 \mathrm{~km}$, 100 from 5 to $10 \mathrm{~km}$ and 100 from $>10 \mathrm{~km}$. The villages were selected using systematic random sampling technique. From each household, all the available family members were interviewed for KAP and saliva samples were collected of each member for estimation of the Snyder test. The selection criteria followed 25 years post implementation was the same as followed and approved by ICMR in 1985 . The changes in KAP about oral health and Snyder test of the adult community of the experimental and control blocks was evaluated using a specially prepared KAP proforma.

\section{Standardization of Personnel}

The social worker was trained in the collection of the KAP data, filling up of the proformas, collection of saliva samples for Snyder test ${ }^{6}$ and how to record the color changes in Snyder media tubes at different time intervals after incubation.

\section{Preparation and Recording of Snyder Test}

Snyder media was prepared and autoclaved in the microbiology department of the institute. Out of the prepared Snyder media tubes, one tube was incubated for 24 hours to check for any microbial growth in order to ensure sterilization of the media. The Snyder tubes were carried into the field area in an icebox for collection of saliva samples from each adult recorded for KAP. The tube was initially rolled between hands to bring its temperature close to the body temperature followed by flaming the rim after opening its cap and drooling unstimulated saliva into it in amount sufficient to cover the upper surface of the media. The rim of the tube was re-flamed and cap replaced.

\section{Transportation and Evaluation of Snyder Tubes}

The tubes were labelled as to the name of the individuals and the date of collection and put into an icebox. At the end of the day, the icebox was transported to the $\mathrm{CHC}$ where the media portion of these tubes containing saliva were divided into four parts and incubated at $37^{\circ} \mathrm{C}$. The color changes occurring within the media after time intervals of $24,48,72$ and 96 hours were noted. If the color changed from green to yellow in $1 / 4$ th portion of the tube, it was marked as $1+$ and accordingly the color change in half portion of the tube was marked as $2+$, in $3 / 4$ th of the tube as $3+$ and full media color change was denoted as $4+$. The readings of the Snyder media were evaluated per person and finally the individual was categorized as having high, moderate, low and very low dental caries activity.

\section{RESULTS}

\section{Oral Hygiene Measures}

At the baseline evaluation (1985), 35.5\% of the community in EA used tooth brush as oral hygiene measure, compared to $34.4 \%$ in the CA. Use of tooth brush increased to $84 \%$ in experimental area and $61 \%$ in control area, 3 years after intervention of oral health education program in experimental area (1990), after which the project staff was withdrawn and the program ran with self rhythm. After a gap of 25 years, it is seen that $96.6 \%$ of community of EA and $85.4 \%$ of the community of CA are using brush for cleaning their teeth. The frequency of twice/ day brushing was seen in $7 \%$ of population at baseline which has increased to $56 \%$ at present in the EA. Similarly $0.8 \%$ of community was brushing twice daily at baseline in the control area compared to $7 \%$ at present (Graph 1). 


\section{Knowledge about Dental Plaque and Calculus}

There was complete ignorance regarding dental plaque and calculus among the population in both EA and CA at baseline. Though the present data show that $70 \%$ population in EA, and $6 \%$ population in CA know about dental plaque. Similarly $39 \%$ of the population of EA now have the knowledge regarding dental calculus which was negligible at base line (Graph 2).

\section{Knowledge about Gum Diseases}

At the base line, knowledge regarding etiology and progress of gum diseases was negligible in both experimental and control areas. The awareness increased significantly in experimental area on both these aspects, 3 years after implementation of the oral health education program. According to the present data, $80 \%$ of the population of the EA and about 22 to $35 \%$ population of the CA is aware about gum diseases (Graph 2).

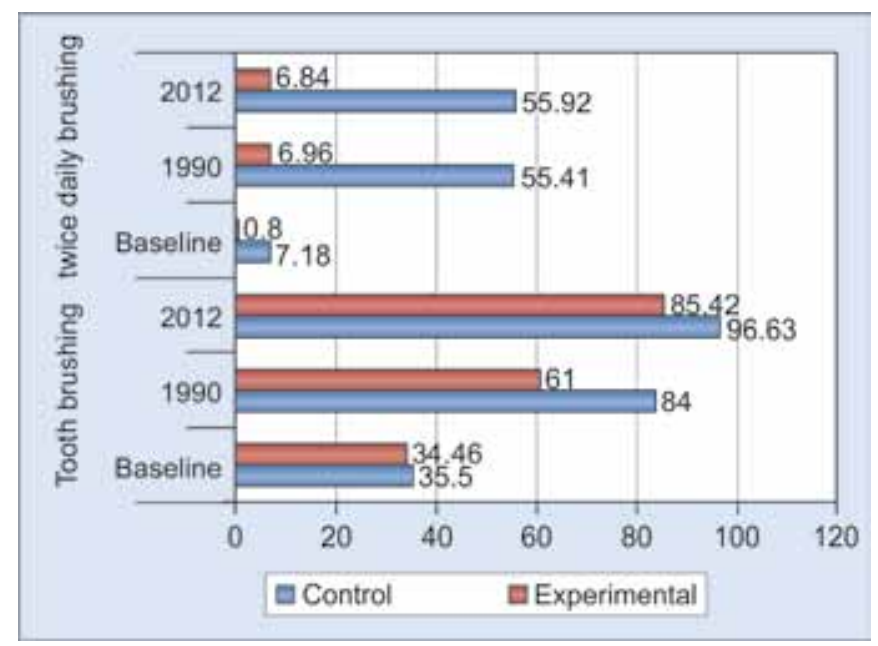

Graph 1: Use of toothbrush as a method of oral hygiene practice among the population

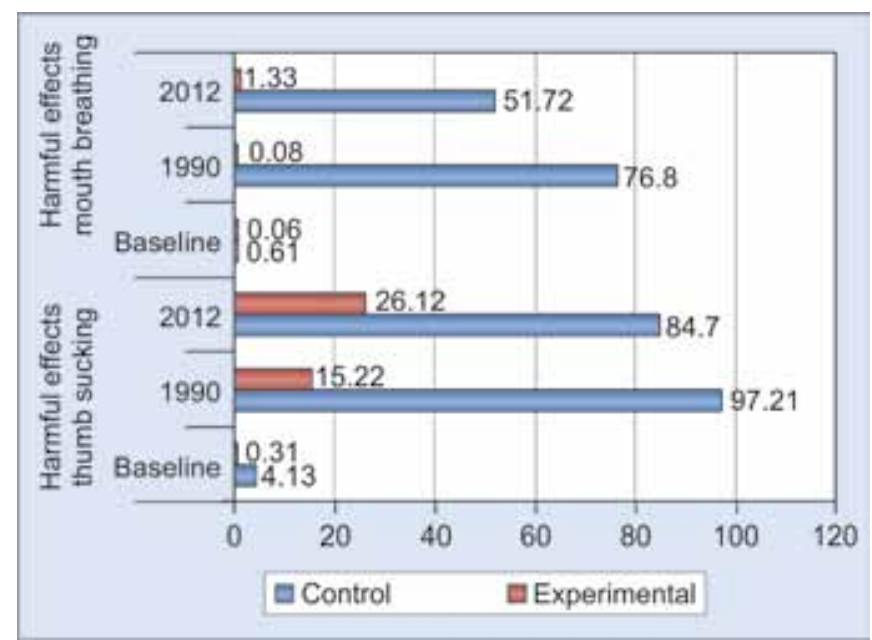

Graph 3: Knowledge about harmful effects of thumb sucking and mouth breathing

\section{Thumb Sucking and Mouth Breathing}

Knowledge regarding harmful effects of thumb sucking was known to only $4 \%$ population of EA and $0.3 \%$ population of the CA at base line. At present, $85 \%$ of the EA population is aware about harmful effects of thumb sucking. Knowledge about harmful effects of mouth breathing was negligible in the community of both EA and CA at the baseline compared to $51 \%$ population of EA and $1.3 \%$ of the CA according to the present data (Graph 3).

\section{Snyder Test Evaluation}

Susceptibility to dental caries has shown a declining trend in EA. Baseline data in 1985 showed that $17 \%$ of the population was at very high risk and $29 \%$ had no risk of developing dental caries. These values have changed to $5 \%$ population at very high risk and $50 \%$ at no risk of developing dental caries 25 years after implementation of the program in the area. The caries activity in CA has

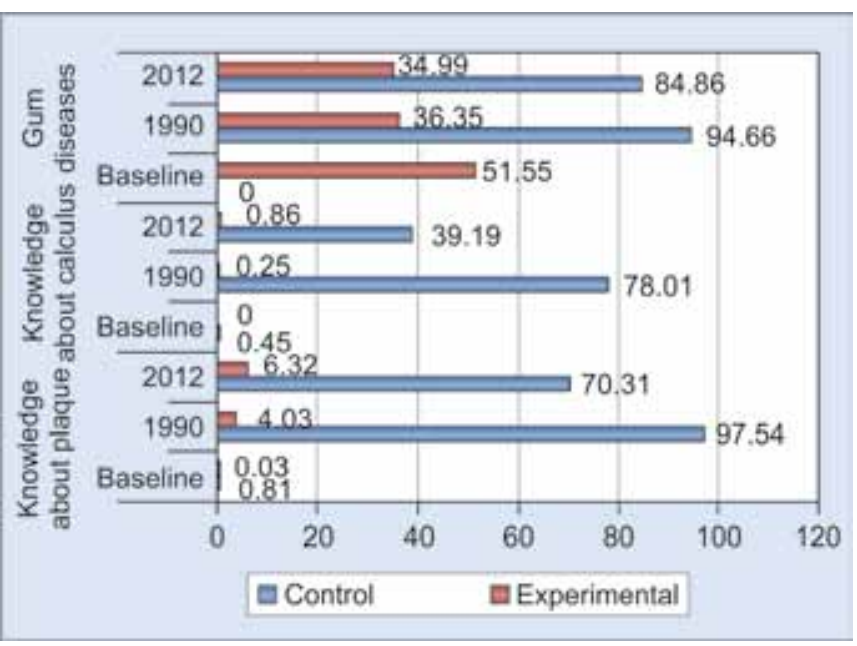

Graph 2: Knowledge about common causes of gum diseases among the community

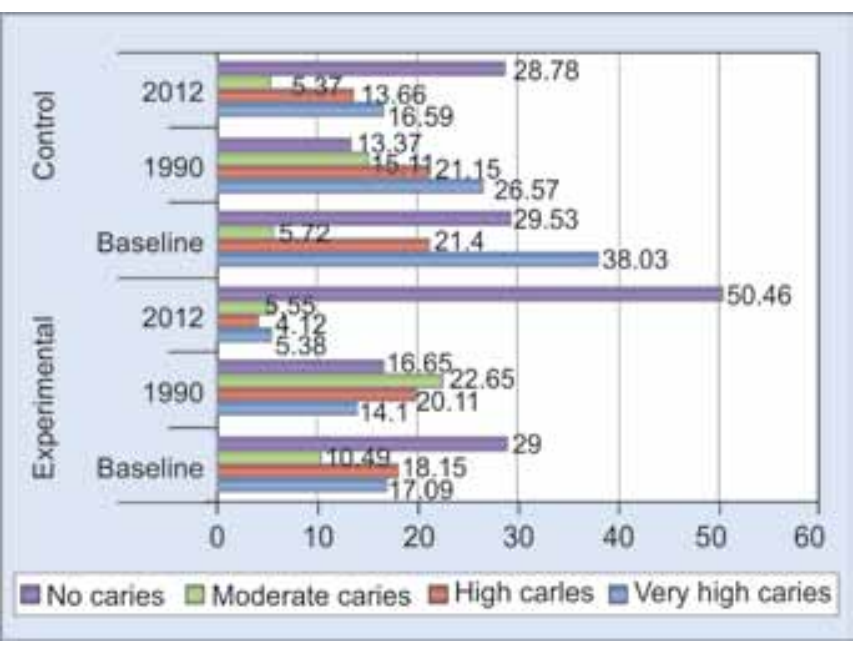

Graph 4: Caries risk among the community as per Snyder test evaluation 
also shown a declining trend but to a lesser extent than the experimental area. At the baseline $38 \%$ had very high caries risk compared to $16 \%$ at present. However, there has been no change in terms of the values observed for no caries risk in the CA population (Graph 4).

\section{DISCUSSION}

The increase in the use of toothbrush in the EA can be attributed to frequent oral health education sessions at village level by the trained health workers, Aanganwadi Workers (AWWs), Auxiliary Nurse Midwife (ANMs), etc. during these 25 years. The knowledge of EA community can be ascribed to the continuous oral health education lectures delivered by the trained health staff during their routine beat program which shows that the program is running by self rhythm. Such a program was however not implemented in the CA. It may be inferred that marginal increase in twice daily brushing frequency of the control area could be due to dissemination of information through TV programs, radio and other means of communication. These findings are in agreement with the findings of John R Greene. ${ }^{7}$ Knowledge about dental plaque and calculus increased among the population of experimental area after the community-based program in 1987 was started but gradually it has deteriorated over the years and needs to be stressed upon even in the experimental area. In the control area, knowledge on this aspect is nonexistent. These findings are in agreement with the study by Tolvalen et al. ${ }^{8}$ Awareness regarding the thumb sucking and mouth breathing was found to be higher in EA compared to CA which may be due to the pictorial description of thumb sucking and its effects on definition as detailed in the oral health education album provided to health workers and school teachers for regular use. In control area, as per the present data, $26 \%$ have this knowledge. Though change in level of knowledge has been demonstrated in this study it may not be feasible to transform it in to behavior every time as per the systematic review by Kay et al. ${ }^{9}$ There was a decrease in prevalence of very high caries risk individuals in the experimental area from 17 to $5 \%$ which may be attributed to improved routine oral hygiene methods, use of fluoridated tooth paste, change in the dietary habits, etc. In a similar study by Vanobbergen $\mathrm{J}$ et al, measurable changes in oral health were not found. ${ }^{10}$

\section{CONCLUSION}

The above data clearly reveals that the health workers in rural areas of Haryana are a viable means of implementing oral health education program in the community. Once trained, they deliver the oral health education to the adult community on a regular basis. The results of the present project clearly emphasize that the oral health education imparted by the trained health workers on a regular basis was retained and practiced by the community even 25 years after the implementation of the oral health education program in the experimental block of Raipur Rani, Haryana.

\section{ACKNOWLEDGMENT}

This research was supported by an ICMR grant to Oral Health Sciences Center, PGIMER, Chandigarh (2011-2014).

\section{REFERENCES}

1. Narayan KMV, Ali MK, Koplan JP. Global non-communicable diseases where worlds meet. N Engl J Med 2010;363(13): 1196-1198.

2. National Oral Health Survey and Fluoride mapping 2002-2003 India, Dental Council of India, New Delhi.

3. Grossi SG, Genco RJ. Periodontal disease and diabetes mellitus: a two-way relationship. Ann Periodontol 1998;3(1): 51-61.

4. Kinane DF. Periodontal diseases contributions to cardiovascular disease: an overview of potential mechanisms. Ann Periodontol 1998;3(1):142-150.

5. Offenbacher S, Katz V, Fertik G, Collins J, Boyd D, Maynor G, et al. Periodontal infection as a possible risk factor for preterm low birth weight. J Periodontol 1996;67(10):1103-1113.

6. Snyder ML. A simple colorimetric method for the estimation of relative numbers of lactobacilli in the saliva. J Dent Res 1940;19:349-355.

7. John RG. Oral health promotion can be effective in changing knowledge. Evidence Based Dentist 1998;1(1):12.

8. Tolvanen M, Lahti S, Hausen H. Changes in tooth brushing frequency in relation to changes in oral health-related knowledge and attitudes among children: a longitudinal study. Eur J Oral Sci 2010;118(3):284-289.

9. Kay E, Locker D. A systematic review of the effectiveness of health promotion aimed at improving oral health. Community Dent Health 1998;15(3):132-44.

10. Vanobbergen J, Declerck D, Mwalili S, Martens L. The effectiveness of a 6-year oral health education programme for primary schoolchildren. Community Dent Oral Epidemiol 2004;32(3):173-182. 